\title{
RESOLUCIÓN NO QUIRÚRGICA DE PIEZA DENTARIA CON LESIÓN PERIAPICAL Y ÁPICE ABIERTO EN PACIENTE ADULTO, TRATAMIENTO CON LÁSER DE DIODO. REPORTE DE CASO.
}

\author{
Non-surgical resolution of a tooth with periapical injury and open apex in an adult \\ patient, laser diode treatment. Case Report.
}

\author{
Laura Beatriz Pinasco $^{1 *}$, Martín Patafio ${ }^{2}$, Ana Casadoumecq ${ }^{3}$, Pablo Alejandro Rodriguez ${ }^{4}$. \\ ${ }^{1}$ Profesora Adjunta Cátedra de Endodoncia Facultad de Odontología, Universidad de Buenos Aires, Argentina. \\ ${ }^{2}$ Docente Cátedra de Endodoncia Facultad de Odontología, Universidad de Buenos Aires, Argentina. \\ ${ }^{3}$ Coordinadora Unidad Láser Facultad de Odontología,Universidad de Buenos Aires, Argentina. \\ ${ }^{4}$ Decano y profesor titular de la Facultad de Odontología, Universidad de Buenos Aires, Argentina. \\ *laura.pinasco@odontologia.uba.ar
}

\section{Resumen}

Los traumatismos dentoalveolares son lesiones que afectan no solo a las piezas dentarias, sino también a los tejidos de sostén. Los mismos pueden traer aparejados diversas secuelas, entre ellas la necrosis pulpar. Si no son detectadas a tiempo puede sumarse una lesión periapical complicando aún más el cuadro clínico. En los casos de dientes permanentes jóvenes, el tratamiento conlleva un desafío en la terapéutica, ya que se produce una interrupción del desarrollo radicular, presentando paredes de poco espesor y ápices abiertos. El siguiente artículo es un reporte de caso clínico de un paciente que concurre a la consulta con antecedente de traumatismo en sector anterior, presentando su incisivo central superior con su anatomía radicular incompleta y con lesión periapical extensa. Su tratamiento incluyó una terapia endodóntica con un cierre apical con Biodentine y aplicación de terapia láser. El seguimiento luego de 24 meses de finalizado el tratamiento, nos muestra al control tomográfico y clínico una evolución favorable. A pesar de presentar una lesión periapical extensa, el tratamiento endodóntico convencional, fue la primera elección.

Palabras clave: Traumatismos, Ápice abierto, Láser diodo, Biodentine ( DeCs).

\begin{abstract}
Dentoalveolar trauma affects not only the teeth but also the supporting tissues. It can be followed by various sequelae, among them pulp necrosis. These if not detected in time, can worsen the clinical picture. In cases of young permanent teeth, the treatment involves a challenge in the therapy, since there is an interruption in root development, causing the tooth to present thin walls and an open apex. The following article is a case report of a patient who presents to the appointment with a history of trauma in the anterior sector, presenting his upper central incisor with incomplete root development and an extensive periapical lesion. The chosen treatment included endodontic therapy with a Biodentine apical plug and application of laser therapy. Follow-up after 24 months of completion of the treatment showed a favorable evolution both in the CBCT and clinical control. Despite presenting extensive periapical injury, conventional endodontic treatment was the first choice.
\end{abstract}

Key words: Trauma, Open apex, Laser diode, Biodentine.

\section{Introducción}

Una de las complicaciones que pueden producirse luego de un traumatismo en una pieza dentaria joven, es la pérdida de la vitalidad pulpar, la cual conduce a la interrupción del desarrollo radicular. Esto plantea un escenario desfavorable, ya que de acuerdo al momento del desarrollo dentario en el que ocurra el traumatismo, ocasionaría que dicha pieza dentaria tenga raíces cortas y paredes radiculares de poco espesor, lo que conlleva un elevado riesgo de fracturas durante la función. ${ }^{1}$ 
A su vez, el tratamiento de los dientes con ápice inmaduro supone un verdadero reto por su dificultad de ejecución, debido a que poseen una anatomía muy accesible para el operador en el examen radiográfico, pero al momento de la obturación no se encuentra una delimitación anatómica que nos de la contención del material dentro del canal radicular, sin poder evitar la extrusión del mismo al espacio periapical.

Las variantes terapéuticas en dientes permanentes jóvenes en caso de requerir tratamiento endodóntico, serían la apicogénesis, en casos de pulpas vitales, la cual permite continuar con el desarrollo radicular tanto en largo como en espesor de las paredes radiculares, y la apicoformación o apexificación, en los casos de necrosis pulpar. ${ }^{2}$

La American Association of Endodontists (A.A.E.) en el glosario de términos endodónticos, define a la apicoformación o apexificación, como un método para inducir una barrera calcificada en una raíz con ápice abierto o con un incompleto desarrollo radicular en dientes con pulpas necróticas. ${ }^{3}$ El objetivo de este tratamiento es obtener una barrera apical para impedir el paso de toxinas y bacterias hacia los tejidos perirradiculares. Técnicamente, esta barrera también es necesaria para permitir la compactación del material de obturación.

Para la apexificación, las opciones de tratamientos en caso de paciente adultos serian: la utilización de medicación intraconducto como hidróxido de calcio $(\mathrm{Ca}(\mathrm{OH}) 2)$, la colocación del agregado de trióxido mineral (MTA) o Biodentine (Septodont, Saint-Maur-desFossés, Francia) como barrera apical antes de obturar el sistema de conductos radiculares en una cita ${ }^{4}$ y la técnica de revascularización. ${ }^{5}$

La utilización de hidróxido de calcio $(\mathrm{Ca}(\mathrm{OH}) 2)$, descripta por Frank $^{6}$ para la apexificación, fue la de mayor elección durante mucho tiempo con un alto índice de resultados favorables, debido a las propiedades antibacterianas que posee, por la liberación de iones hidroxilos, los cuales alteran la integridad de la membrana citoplasmática bacteriana ${ }^{7}$ y gracias a su alcalinidad $(\mathrm{pH}$ 12,5) que actúa como un factor aparente en la inducción de la calcificación apical. Sin embargo, presenta desventajas tales como: el largo tiempo de tratamiento necesario para obtener la barrera calcificada, que puede variar entre 6 a 24 meses; la necesidad de múltiples renovaciones, las cuales pueden traer aparejadas contaminaciones; numerosas citas superditadas a la predisposición del paciente; la imprevisibilidad de cierre apical, microfiltración y el debilitamiento de las ya frágiles paredes de la raíz, como resultado de las renovaciones. ${ }^{8}$

En la actualidad, con el fin de evitar estas desventajas, la terapia de apexificación con el hidróxido de calcio está siendo desplazado por el uso de materiales bioceramicos como el MTA y Biodentine ${ }^{\mathrm{tm}}$ en la técnica de un solo paso en determinadas situaciones clínicas. ${ }^{9,10}$ Numerosos autores como Witherspoon y Ham 2001, Linsuwanont 2003, Andreasen y cols., 2006, han descripto esta técnica de obturación con un tapón apical de material biocerámico en los últimos $5 \mathrm{~mm}$ del conducto en una sola cita.

Ambos materiales, tanto el MTA como el Biodentine ${ }^{\mathrm{tm}}$, presentan similares características en cuanto a biocompatilidad. El Biodentine ${ }^{\mathrm{tm}}$ es un cemento bioactivo a base de silicato de calcio, con características de resistencia y módulo de elasticidad similar a la dentina. Otra ventaja sobre el MTA es el rápido fraguado, obteniendo su máxima dureza luego de 24 horas, ${ }^{11-14}$ y a su vez posee menor riesgo de pigmentación. ${ }^{15}$ Estas características, lo convierte en un material de primera elección para tratamientos de apexificación.

La preparación de los conductos consta de 2 ejes principales la conformación mecánica y la preparación química. Ambos tienen como objetivo lograr una superficie apta para ser obturada. El Enterococcus faecalis, rara vez se encuentra en grandes proporciones en la raíz sin tratar, pero están altamente asociados con fallas en los tratamientos de conducto. Cuando las bacterias penetran en las capas profundas de la dentina, la desinfección de los túbulos dentinarios se vuelve más difícil. Los irrigantes existentes eliminan microorganismos a través del contacto directo con ellos y, por lo tanto, no pueden penetrar profundamente en los tubos de dentina y limpiarlos. ${ }^{16}$ Para favorecer la desinfección del conducto se puede utilizar láser de diodo.

Uno de los principales objetivos del tratamiento endodóntico es dejar el conducto radicular libre de microorganismos. Para ello se pueden utilizar diferentes sistemas de desinfección y el láser de diodo es uno de ellos. No obstante, se debe de tener precaución con su uso a fin de prevenir el posible daño térmico generado por el láser sobre el periodonto o el propio diente. ${ }^{17}$

El láser de diodo es poco absorbido por las estructuras dentales, el efecto térmico de la radiación se transmite en profundidad a través de la dentina con una baja interacción con él, lo que permite un efecto foto disruptivo sobre los microorganismos presentes en las partes inalcanzables de la red tubular. ${ }^{18}$

El objetivo de este trabajo es presentar un caso clínico con un control a dos años de un tratamiento de apexificación mediante un tapón apical de Biodentine ${ }^{\mathrm{tm}}$ conjunto a una terapéutica láser en una pieza dentaria con un cierre apical incompleto y una lesión periapical extensa. 


\section{Presentación del caso}

Se presentó a la consulta un paciente masculino de 20 años, derivado de la Cátedra de Ortodoncia por poseer una lesión periapical en pieza dentaria 2.1. Al examen clínico (figura 1) se observó una fistula activa con exudado purulento, ausencia de sintomatología dolorosa y movilidad leve en dicha pieza, la cual respondió negativamente al test de sensibilidad pulpar al frío. Al examen radiográfico (figura 2), la pieza dentaria 2.1 presentaba su ápice radicular sin desarrollar con una lesión radiolúcida periapical, en íntima relación con la pieza dentaria 2.2.

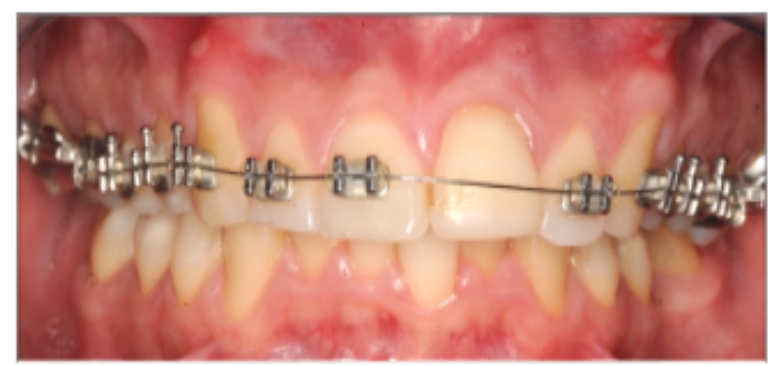

Fig. 1. Examen clínico inicial

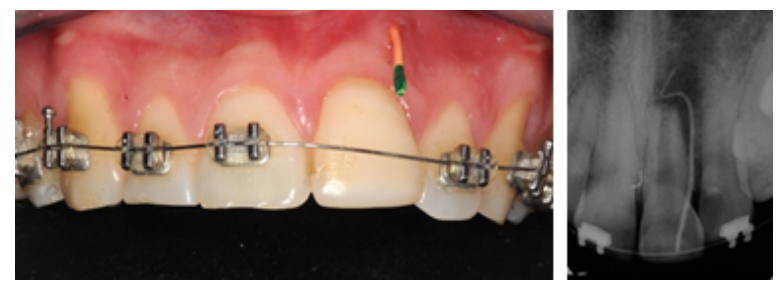

Fig. 2. Examen radiográfico y fistulografía

Al examen tomográfico inicial se observó la pieza dentaria 2.1 con una radiolucidez apical, ausencia de tabla vestibular y con un incompleto cierre del ápice radicular. (figura 3)
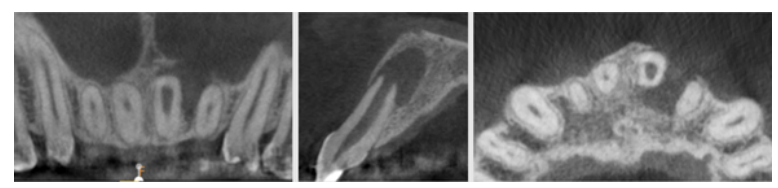

Fig. 3. Examen tomográfico inicial

El paciente relató recordar un traumatismo producto de una caída a los 6 años de edad con fractura del borde incisal de dicha pieza, sin atención odontológica de urgencia inmediata. La reconstrucción estética con resina compuesta fue realizada a las semanas del traumatismo.
Luego de evaluar las opciones terapéuticas, se decidió llevar a cabo un tratamiento no quirúrgico de apexificación; se informó al paciente que, de no observar una evolución correcta o una remisión total de la lesión, se complementaría el tratamiento con microcirugía apical de dicha pieza dentaria.

En la primera sesión se realizó la apertura, se colocó hidróxido de calcio intraconducto, ya que presentaba persistencia de drenaje apical, y se realizó un doble sellado coronario con teflón estéril y ionómero vitreo de autocurado. (Figura 4)

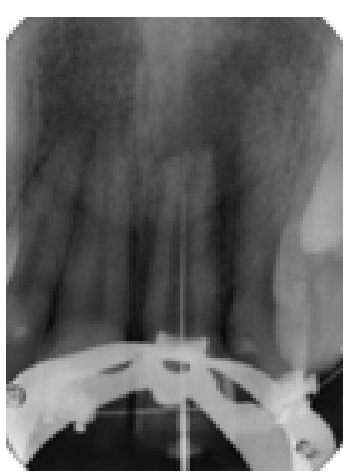

(a) Conductometría

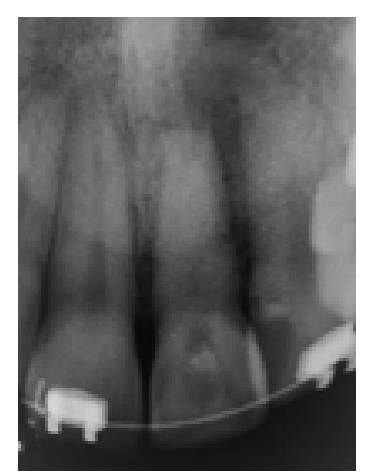

(b) Radiografía luego de la colocación de hidróxido de calcio.
Fig. 4. Primera sesión

En la segunda sesión, luego de 15 días, al observar persistencia de actividad en la fistula ,se optó por complementar el tratamiento con láser terapia. Con el fin de lograr una remoción lo más efectiva posible del hidróxido de calcio, la preparación química se complementó con la utilización de la lima XP-Endo Finisher ® durante 60 segundos, a una velocidad de 800 r.p.m y $1 \mathrm{NCm}$ de torque, ya que en varios estudios se comprobó su efectividad en la remoción de medicación intraconducto. ${ }^{19-21}$

Luego se utilizó láser diodo de 940nm (Biolase®) con un tip endodóntico estéril, de 200um a 1 watt de potencia en 4 ciclos de $2 \mathrm{~mm} / \mathrm{seg}$ según la longitud de trabajo intracanal, para la descontaminación de las paredes dentinarias a posterior de la instrumentación y lavajes del conducto. Se utilizó además intrafistula, para la descontaminación de la zona del tejido de degranulación y la desepitelialización de la fistula. (figura 5)

En esa misma sesión, se realizó un tapón apical con Biodentine ${ }^{\mathrm{tm}}$ (Septodont) de aproximadamente 4 $\mathrm{mm}$ de longitud, colocándolo con limas y realizando una compactación vertical con limas emboladas estériles y atacadores Maillefer ${ }^{\circledR}$ de Dentsply números 2-4. Se 


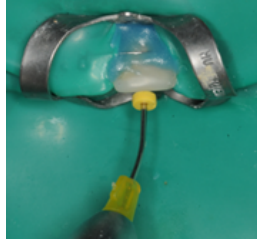

(a) Terapia laser intracanal
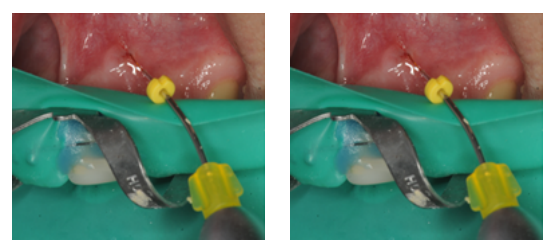

fistula (c) Resultado inmediato

Fig. 5. Segunda sesión

finalizó la obturación con gutapercha con impresión apical y técnica hibrida de Tagger, y luego se realizó la restauración definitiva con resina Brillianttm NG de Coltene para asegurar el sellado coronario. (figura 6) Se indicó medicación antibiótica amoxicilina $500 \mathrm{mg}$ cada 8 horas por 7 días.

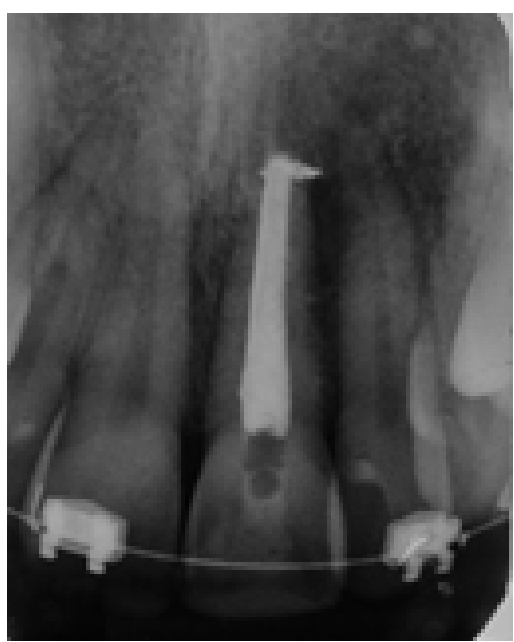

Fig. 6. Tapón apical

Se realizó un control al mes de realizado el tapón apical de Biodentine ${ }^{\mathrm{tm}}$, en el cual el paciente no presentó sintomatología clínica ni particularidades a nivel radiográfico (figura 7).
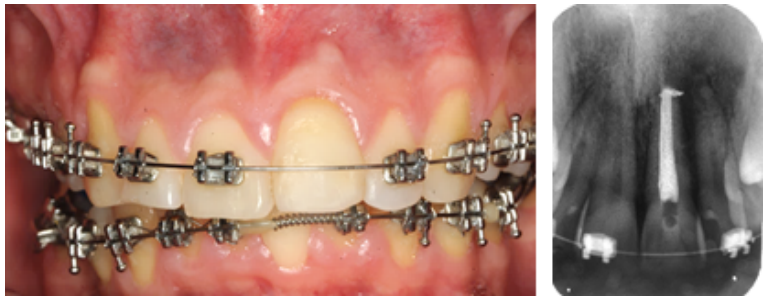

Fig. 7. Revisión al mes

A los 6 meses de finalizado el tratamiento, se solicitó una CBCT en la cual se observó una marcada disminución de la zona radiolúcida y una pequeña extrusión de Biodentine ${ }^{\mathrm{tm}}$ en la zona de la lesión periapiapical (figura 8).
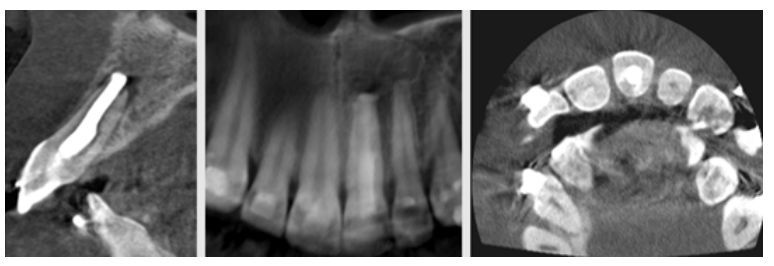

Fig. 8. Revisión a los 6 meses

A los 18 meses de finalizado el tratamiento, se realizó un control clínico (figura 10) y tomográfico (figura 9). En la tomografía se observó una marcada disminución de la imagen radiolúcida periapical, pudiendo distinguir una línea del ligamento periodontal continua y conservación de la tabla ósea vestibular. A partir de este momento, se autorizó a reanudar su tratamiento ortodóncico.
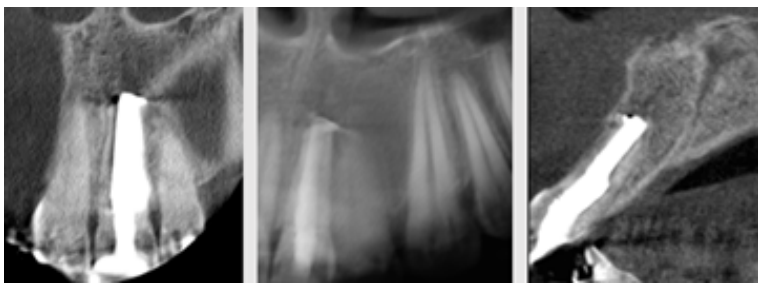

Fig. 9. Control tomográfico a los 18 meses de iniciado el tratamiento

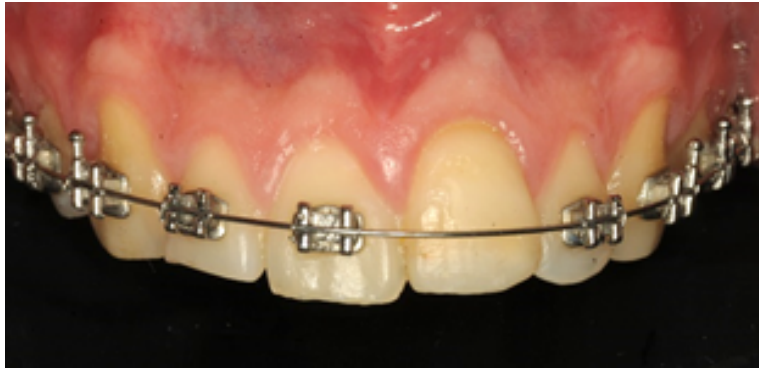

Fig. 10. Control tomográfico a los 18 meses de iniciado el tratamiento

Se realizó un nuevo control clínico y tomográfico a los 24 meses de finalizado el tratamiento. Clínicamente se puede observar la salud de tejidos periodontales y una buena respuesta de la pieza dentaria 2.1 al tratamiento ortodóntico. (Figura 11). Al examen tomográfico se observó una curación acorde a los controles anteriores y buena respuesta de los tejidos a las fuerzas ortodónticas (Figura 12). 


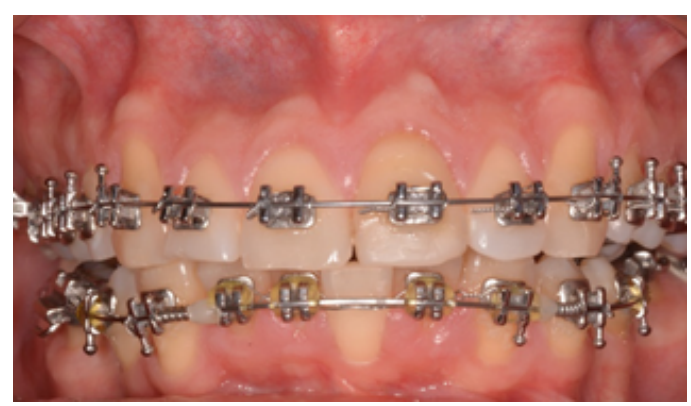

(a) Control clínicol
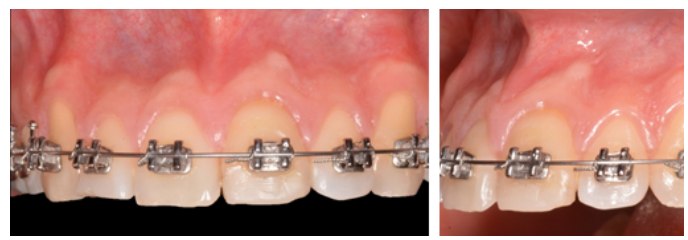

(b) Control clínico

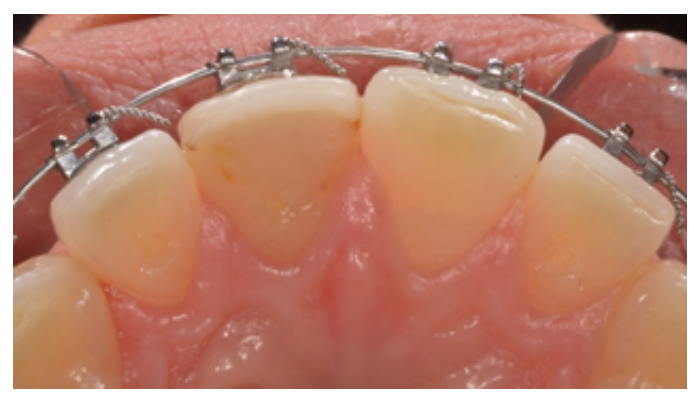

(c) Control clínico

Fig. 11. 24 meses de finalizado el tratamiento
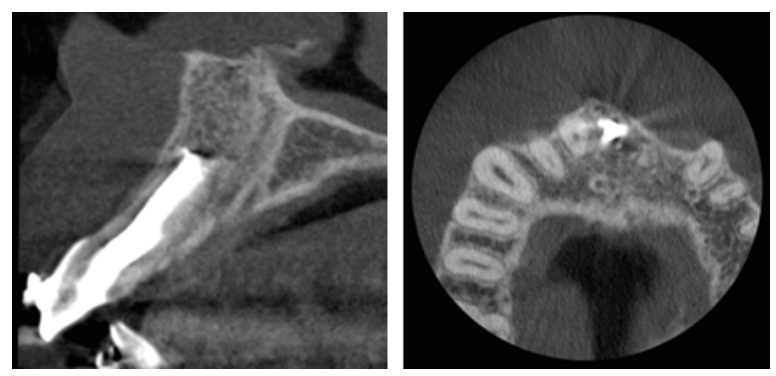

Fig. 12. Examen tomográfico

\section{Discusión}

Durante muchos años, la apexificación en dientes permanentes jóvenes con necrosis, se ha realizado mediante la utilización de $\mathrm{Ca}(\mathrm{OH}) 2$ con protocolos de recambio hasta lograr el cierre apical, pero esta técnica conlleva ciertas desventajas que incluyen: la variabilidad en el tiempo del tratamiento, la dificultad en el seguimiento de los pacientes y el riesgo aumentado de fractura radicular a nivel cervical por el uso de este material en periodos de tiempo prolongados, ${ }^{22}$ ya que su alcalinidad desnaturaliza las proteínas de la matriz orgánica de la dentina, ${ }^{23}$ el que lo convierte en un material de no elección hoy en día.

Varios trabajos, como el de Prahan et al., ${ }^{24}$ manifiesta la eficacia del MTA cuando se utiliza en procedimientos de apexificación, y se lleva a cabo una comparación en el tratamiento de dientes con rizogénesis incompleta utilizando $\mathrm{Ca}(\mathrm{OH}) 2$ o MTA. Este dio como resultado que el tiempo total del tratamiento y el tiempo medio necesario para la formación de la barrera biológica fue mucho menor en los pacientes tratados con MTA. A pesar de las muchas ventajas que nos proporcionan los trabajos, el MTA también tiene algunas limitaciones al no generar un refuerzo de las paredes dentinarias del conducto radicular, su alto costo, un tiempo de fraguado alto y la ausencia de un disolvente conocido en caso de ser necesaria su remoción. Así es como Hassan et al., ${ }^{25}$ reportaron la superioridad de Biodentine ${ }^{\mathrm{tm}}$ en comparación con ProRoot MTA en cuanto a la manipulación, velocidad de fraguado y menor microfiltración. ${ }^{26}$

Para asegurar la correcta descontaminación del conducto y de la lesión fue que se llevó a cabo la utilización del láser, ya que este posee una acción antibacterial fototérmica y de fotoactivacion que llega a mayor profundidad en los túbulos dentinarios y a zonas donde el irrigante no puede acceder, además de no ser dañina para los tejidos periodontales. ${ }^{27}$ A fin de eliminar la fístula del tracto tan suavemente como sea posible, la fibra flexible, emite el láser radial, se inserta desde el exterior y se coloca exactamente usando la luz guía. La energía se emite dentro de la fístula. La epitelización del tejido se destruye de una manera controlada y la fístula del tracto colapsa. Esto también acelera el proceso de recuperación. Tal como se plasma en la literatura, la acción terapéutica del láser se potencia con presencia del hipoclorito de sodio, otorgándole mayor poder antibacterial. ${ }^{28,29}$

A pesar de haber elegido una terapéutica de apicoformación, la cual no refuerza la raíz dentaria, esta nos da un tiempo corto de tratamiento con la posibilidad de restaurar inmediatamente el diente sin efectos adversos sobre las propiedades mecánicas de las paredes radiculares. ${ }^{30}$

Estas ventajas, sumadas a la utilización de materiales biocompatibles, la transforma en una técnica predecible y fiable, ya que como se observa en este caso clínico, la extrusión de Biodentine ${ }^{\mathrm{tm}}$ al espacio periapical no ha generado complicaciones en el tratamiento. A pesar de no ser recomendable, numerosos estudios a lo largo del tiempo han demostrado que esto no afecta la curación de los tejidos periapicales. $^{31}$ 


\section{Conclusión}

La apexificación, en uno o dos citas según amerite el caso clínico, con el uso de un tapón apical con materiales biocerámicos como el Biodentine ${ }^{\text {tm }}$, está demostrado que logra un éxito clínico, radiográfico y tomográfico, por lo cual podría ser considerada una buena opción de elección en el tratamiento de piezas permanentes con ápices abiertos y necrosis pulpares en pacientes adultos.

El láser de diodo de $940 \mathrm{~nm}$ puede ser un complemento exitoso al tratamiento endodóntico convencional de casos necróticos con lesiones periapicales crónicas en términos de desinfección del conducto radicular y despitelización del trayecto fistuloso.

Conflicto de intereses: Los autores del presente estudio manifiestan que no existe ningún conflicto de intereses en relación al tema de estudio.

\section{Referencias}

1 Kenneth M. Hargreaves, DDS, PhD, Anibal Diogenes, DDS, MS, PhD, and Fabricio B. Teixeira, DDS, PhD. Treatment Options: Biological Basis of Regenerative Endodontic Procedures. J Endod. 2013 Mar; 39(3 Suppl): S30-S43.

2 Shabahang S. Retreatment options: apexogenesis and apexification. J Endod. 2013 Mar;39(3 Suppl):S26-9

3 American Association of Endodontists (A.A. E.). Glosary of tenns used in endodontics. 4th Edición, 1984.

4 Frank A. Therapy for divergent pulpless tooth by continued apical formation. J Am Dent Assoc. 1966; 72(1):87-93.

5 Jeeruphan T, Jantarat J, Yanpiset K, et al. Mahidol study 1: comparison of radiographic and survival outcomes of immature teeth treated with either regenerative endodontic or apexification methods: a retrospective study. J Endod. 2012; 38(10):1330-1336

6 Frank A. Therapy for divergent pulpless tooth by continued apical formation. J Am Dent Assoc. 1966; 72(1):87-93.

7 Estrela C ; Sydney GB, Bammann LL, Felippe Júnior O. Mechanism of Action of Calcium and Hydroxyl Ions of Calcium Hydroxide on Tissue and Bacteria. Braz Dent J (1995) 6(2): 85-90 ISSN 0103-6440

8 Andreasen JO, Farik B, Munksgaard EC. Long-term calcium hydroxide as a root canal dressing may increase risk of root fracture. Dental Traumatol. 2002; 18(3):134- 137.

9 Guerrero F, Mendoza A, Ribas D, Aspiazu K. Apexification: a systematic review. J Conserv Dent. 2018 Sep-Oct; 21(5): 462-465

10 Khetarpal A, Chaudhary S, Talwar S, Verma M. Endodontic management of open apex using Biodentine as a novel apical matrix. Indian J Dent Res 2014;25:513-6

11 About I. Biodentine: from biochemical and bioactive properties to clinical applications. G Ital Endod. 2016; 30: 8188
12 Nayak G, Hasan MF. Biodentine-a novel dentinal substitute for single visit apexification. Restor Dent Endod. 2014 May;39(2):120-125.

13 Sinkar RC, Patil SS, Jogad NP, Gade VJ. Comparison of sealing ability of ProRoot MTA, RetroMTA, and Biodentine as furcation repair materials: An ultraviolet spectrophotometric analysis. J Conserv Dent. 2015;18(6):445-448.

14 Bajwa NK, Jingarwar MM, Pathak A. Single Visit Apexification Procedure of a Traumatically Injured Tooth with a Novel Bioinductive Material (Biodentine). Int J Clin Pediatr Dent. 2015;8(1):58-61.

15 Kaur, M., Singh, H., Dhillon, J. S., Batra, M., \& Saini, M. (2017). MTA versus Biodentine: Review of Literature with a Comparative Analysis. Journal of Clinical and Diagnostic Research: JCDR, 11(8), ZG01

16 Basualdo, Juan Angel - Coto, Celia E. - Torres, Ramón Alberto de Microbiología biomédica : bacteriología - micología - virología - parasitología - inmunología. - 2a ed.. Buenos Aires : Atlante, 2006. xxviii, 1537 p. : il., fotos ; 28 cm. ISBN: 950-9539-47-3978-950-9539-47-1

17 Larrea-Oyarbide, Nerea, España-Tost, Antonio Jesús, Berini-Aytés, Leonardo, \& Gay-Escoda, Cosme. (2004). Aplicaciones del láser de diodo en Odontología. RCOE, 9(5), 529-534.

18 López-Jiménez L1, Arnabat-Domínguez J, Viñas M, Vinuesa T. Atomic force microscopy visualization of injuries in Enterococcus faecalis surface caused by Er,Cr:YSGG and diode lásers. Med Oral Patol Oral Cir Bucal. 2015 Jan 1;20(1):e45-51

19 Wigler R, Dvir R, Weisman A, Matalon S, Kfir A. Efficacy of XP-endo finisher files in the removal of calcium hydroxide paste from artificial standardized grooves in the apical third of oval root canals. International Endod J. 2017. 50 (7), 700-705.

20 Hamdan R, Michetti J, Pinchon D, Diemer F, GeorgelinGurgel M. The XP-Endo Finisher for the removal of calcium hydroxide paste from root canals and from the apical third. J Clin Exp Dent. 2017;9(7):e855-e860. Published 2017 Jul 1. doi:10.4317/jced.53962

21 CangülKeskin, EvrenSariyilmaz, ÖznurSariyilmaz. Efficacy of XP-endo Finisher File in Removing Calcium Hydroxide from Simulated Internal Resorption Cavity. Journal of Endodontics. 2017. 43(1), 126-130.

22 Cvek M. L'hydroxyde de calcium dans le traitement des dents traumatisées. Rev Fran Endod1989;8:11-27.

23 Andreasen JO, Farik B, Munksgaard EC. Long-term calcium hydroxide as a root canal dressing may increase risk of root fracture. Dent Traumatol. 2002;18:134-7.

24 Pradhan DP, Chawla HS, Gauba K, et al. Comparative evaluation of endodontic management of teeth with unformed apices with MTA and calcium hydroxide. J Dent Child. 2006; 73(2):79-85

25 Hassan F, Al Hadi D, MH S. Furcal perforation repair using MTA \& Biodentine, an in vitro evaluation using dye 
extraction method. Int J Recent Sci Res. 2015; 6(3): 31723175

26 Allwyn S, Sharath A, Seby T. Evaluation of sealing ability of Biodentine and mineral trioxide aggregate in primary molars using scanning electron microscope: A randomized controlled in vitro trial. Contemp ClinDent. 2016; 7(3): 322-325.

27 A. Namour, S. Geerts, T. Zeinoun, R. De Moor, and S. Nammour, "Safety Irradiation Parameters of Nd:YAP Laser Beam for Endodontic Treatments: An In Vitro Study," BioMed Research International, vol. 2016, Article ID 4741516, 5 pages, 2016

28 Katalinić I, Budimir A, Bošnjak Z, Jakovljević S, Anić I. The photo-activated and photo-thermal effect of the 445/970 $\mathrm{nm}$ diode laser on the mixed biofilm inside root canals of human teeth in vitro: A pilot study. Photodiagnosis Photodyn Ther. 2019 Jun;26:277-283.

29 Asnaashari M, Safavi N. Disinfection of Contaminated Canals by Different Laser Wavelengths, while Performing Root Canal Therapy. J Lasers Med Sci. 2013;4(1):8-16.

30 Simon S, Rilliard F, Berdal A, Machtou P. The use of mineral trioxide aggregate in one-visit apexification treatment: A prospective study. Int Endod J. 2007;40:186-97.

31 Bani M, Sungurtekin-Ekçi E, Odabaş ME. Efficacy of Biodentine as an Apical Plug in Nonvital Permanent Teeth with Open Apices: An In Vitro Study. Biomed Res Int. 2015;2015:359275.

Recibido: 22 de marzo de 2021

Aceptado: 01 de mayo de 2021 
